\title{
Generalised finite volume strategies for simulating transport in strongly orthotropic porous media
}

Jayantha Pasdunkorale A.* $\quad$ Ian W. Turner ${ }^{\dagger}$ (17 July 2001)

\begin{abstract}
In this work two different finite volume computational strategies for solving a representative two-dimensional diffusion equation in an orthotropic medium are considered. When the diffusivity tensor is treated as linear, this problem admits an analytic solution used for analysing the accuracy of the proposed numerical methods. In the first method, the gradient approximation techniques discussed by Jayantha and Turner [Numerical Heat Transfer, Part B: Fundamentals, 40, pp.367-390, 2001] are applied directly to the
\end{abstract}

*School of Mathematical Sciences, Queensland University of Technology, Australia. On leave from University of Ruhuna, SRI Lanka. mailto: jayanthapa@yahoo.com, pa.jayantha@fsc.qut.edu.au

${ }^{\dagger}$ School of Mathematical Sciences, Queensland University of Technology, Australia mailto:i.turner@qut.edu.au

${ }^{0}$ See http://anziamj. austms.org. au/V44/CTAC2001/Jaya for this article, (C) Austral. Mathematical Soc. 2003. Published 1 April 2003. ISSN 1446-8735 
diffusion equation. In the second method, the diffusion equation is transformed via scaling parameters to an isotropic model and then the control volume techniques discussed by Jayantha and Turner are used to obtain the numerical results on the transformed domain. Both methods are shown to produce reasonable results in comparison with the exact solution for a range of anisotropy ratios typical of wood. However, only the first method is appropriate for use in nonlinear coupled transport systems. This work highlights the necessity of determining a higher order gradient approximation to improve the numerical results on the untransformed domain.

\section{Contents}

1 Introduction

C445

2 Theoretical Solution

C447

3 Control Volume Technique

C448

3.1 Flux decomposition using vectors (FDV) . . . . C C450

3.1.1 Boundary conditions . . . . . . . . . . C453

3.2 Hybrid CV-FE formulation . . . . . . . . . . . C C454

3.2.1 Boundary Conditions . . . . . . . . . . . C455

4 Transformation into an Isotropic Model $\quad$ C456

5 Numerical Results $\quad$ C457

6 Conclusions $\quad$ C461

References

C462 


\section{Introduction}

The approximation of the flux term is an important issue that needs to be addressed when implementing unstructured mesh finite volume methods for simulating diffusion in strongly orthotropic porous media. Several finite volume discretisation strategies have been proposed for resolving mainly isotropic problems [2, 3, 4, 5, 8, e.g.]. The aim of this work is to analyse various flux approximation schemes to assess their applicability to orthotropic porous media. In particular, the inclusion of the important tangential, or cross diffusion term is investigated for use in a completely generalised finite volume methodology.

In order to achieve this objective, the following two-dimensional orthotropic diffusion problem for a finite rectangular domain is considered:

$$
\nabla \cdot(K \nabla \phi)=\rho C_{p} \frac{\partial \phi}{\partial t}, \quad 0 \leq x \leq L, \quad 0 \leq y \leq M, \quad t>0,
$$

where $K=\operatorname{diag}\left(k_{x x}, k_{y y}\right)$ and

$$
(K \nabla \phi) \cdot \hat{\mathbf{n}}_{b}=h_{b}\left(\phi_{s}-\phi\right) \quad \text { at boundary } S_{b}, \quad t>0,
$$

where $\hat{\mathbf{n}}_{b}$ is the outward unit normal vector. Initially $\phi(x, y, 0)=\phi_{0}$ for $0<x<L$ and $0<y<M$. 


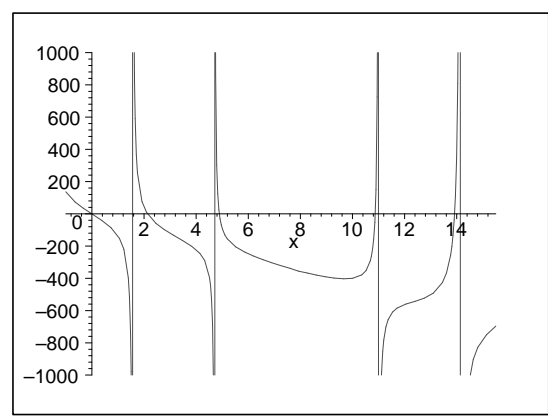

(a)

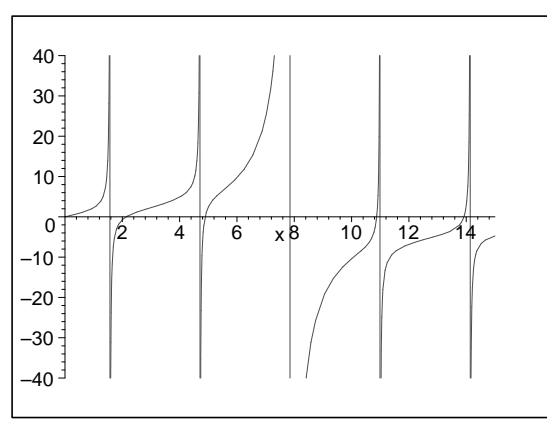

(b)

FiguRE 1: The graphs of: (a) $\left(\mu^{2}-B i_{1} B i_{3}\right) \tan \mu-\left(B i_{1}+B i_{3}\right) \mu$; and (b) $\tan \mu-\left(B i_{1}+B i_{3}\right) \mu /\left(\mu^{2}-B i_{1} B i_{3}\right)$; using $B i_{1}=41.0$ and $B i_{3}=1.5$ over the interval $[0,5 \pi]$. Note that the horizontal axis represents $\mu$ in these graphs and the eigenvalues of equation (3) within the interval are to be seen at the points where the function intersects this axis. 


\section{Theoretical Solution}

To obtain the exact solution, Equation (1) is transformed into dimensionless form by using the following parameters, see also [6, 10]:

$$
\begin{array}{r}
X=\frac{x}{L}, \quad Y=\frac{y}{M}, \quad \tau=\frac{k_{x x} t}{\rho C_{p} L^{2}}, \quad B i_{1}=\frac{h_{1} M}{k_{y y}}, \\
B i_{2}=\frac{h_{2} L}{k_{x x}}, \quad B i_{3}=\frac{h_{3} M}{k_{y y}}, \quad B i_{4}=\frac{h_{4} L}{k_{x x}}, \\
\Theta(X, Y, \tau)=\frac{\left(\phi-\phi_{s}\right)}{\left(\phi_{0}-\phi_{s}\right)}, \quad 0 \leq \Theta \leq 1 .
\end{array}
$$

Then, equation (1) becomes

$$
\frac{\partial^{2} \Theta}{\partial X^{2}}+\frac{k_{y y} L^{2}}{k_{x x} M^{2}} \frac{\partial^{2} \Theta}{\partial Y^{2}}=\frac{\partial \Theta}{\partial \tau} ; \quad 0 \leq X, Y \leq 1, \quad \tau>0,
$$

with boundary conditions for $\tau>0$ of

$$
\begin{array}{cc}
-\frac{\partial \Theta}{\partial X}+B i_{4} \Theta=0 \text { on } X=0, & \frac{\partial \Theta}{\partial X}+B i_{2} \Theta=0 \text { on } X=1 ; \\
-\frac{\partial \Theta}{\partial Y}+B i_{1} \Theta=0 \text { on } Y=0 ; & \frac{\partial \Theta}{\partial Y}+B i_{3} \Theta=0 \text { on } Y=1 ;
\end{array}
$$

and initial condition $\Theta(X, Y, 0)=1$, for $0<X, Y<1$.

Assuming the separation $\Theta(X, Y, \tau)=\Theta_{1}(X, \tau) \Theta_{2}(Y, \tau)$, it can be shown that, for example,

$$
\Theta_{2}(Y, \tau)=\sum_{n=1}^{\infty} \frac{\chi\left(\mu_{n}, Y\right)}{N_{y}\left(\mu_{n}\right)}\left(\sin \mu_{n}-\frac{B i_{1}}{\mu_{n}} \cos \mu_{n}+\frac{B i_{1}}{\mu_{n}}\right) e^{-\mu_{n}^{2} r^{2} \tau}
$$

where $r^{2}=k_{y y} L^{2} /\left(k_{x x} M^{2}\right)$, and

$$
\chi\left(\mu_{n}, Y\right)=\mu_{n} \cos \left(\mu_{n} Y\right)+B i_{1} \sin \left(\mu_{n} Y\right) \quad \text { for } \quad 0<Y<1 ;
$$




$$
\begin{gathered}
\left(\mu_{n}^{2}-B i_{1} B i_{3}\right) \tan \mu_{n}=\left(B i_{1}+B i_{3}\right) \mu_{n} ; \\
N_{y}\left(\mu_{n}\right)=\frac{1}{2}\left[\left(\mu_{n}^{2}+B i_{1}^{2}\right)\left(1+\frac{B i_{3}}{\mu_{n}^{2}+B i_{3}^{2}}\right)+B i_{1}\right] .
\end{gathered}
$$

An example for the roots of the transcendental equation (3) is exhibited in Figure 1. A globally convergent Newton scheme [7] is used to find the desired roots. Using the above equations, the analytic solution of equation (1) for the orthotropic case can be written as

$$
\phi(x, y, t)=\phi_{s}+\Theta_{1}(X, \tau) \Theta_{2}(Y, \tau)\left(\phi_{0}-\phi_{s}\right)
$$

for $0<x<L, 0<y<M, t>0$.

\section{Control Volume Technique}

To construct the control volume mesh, the computational domain is tessellated with triangles and the control volumes are constructed around the vertices of these elements by joining the centroids of adjacent cells. Figure 2(a) exhibits a typical control volume within an unstructured mesh of the cell-centered control volume approach. The control volume with the centroid $P$ has nodes $N_{k}, k=1,2, \ldots, p$. The discretised form of the differential equation (1) is derived by integrating the equation over the control volume $\delta V_{P}$ and in time from $n \delta t$ to $(n+1) \delta t$ :

$$
\begin{gathered}
\rho C_{p} \frac{\delta V_{P}}{\delta t}\left(\phi_{P}^{(n+1)}-\phi_{P}^{(n)}\right)-\lambda \sum_{k=1}^{N p}\{(K \nabla \phi) \cdot \hat{\mathbf{n}}\}_{F_{k}}^{(n+1)} A_{k} \\
-(1-\lambda) \sum_{k=1}^{N p}\{(K \nabla \phi) \cdot \hat{\mathbf{n}}\}_{F_{k}}^{(n)} A_{k} \simeq 0
\end{gathered}
$$




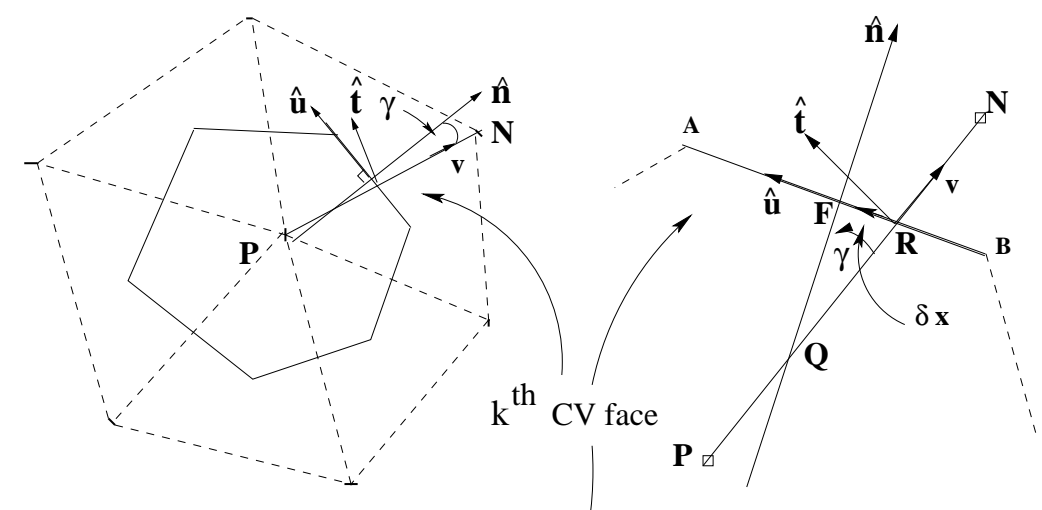

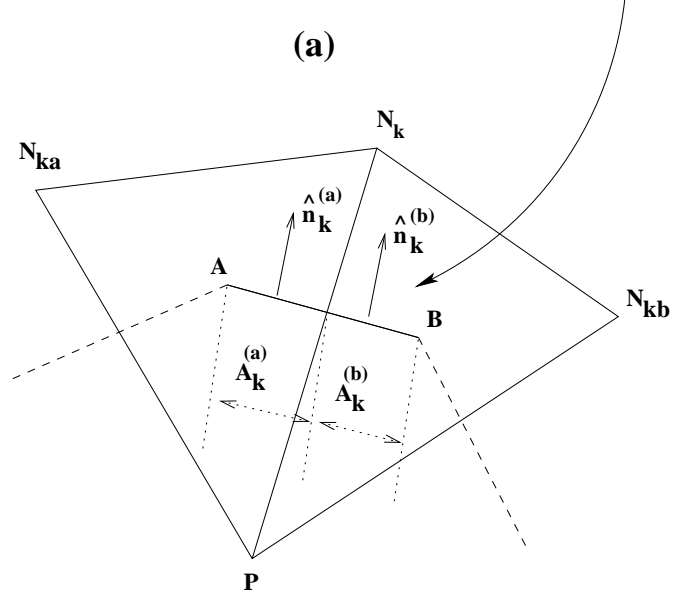

(c)

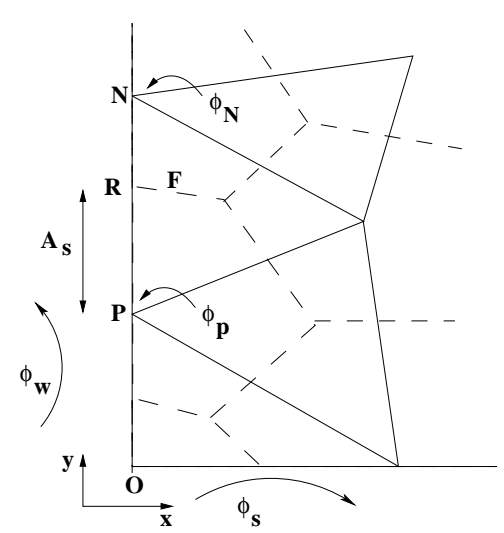

(d)

Figure 2: (a) A control volume (Cv). (b) A CV face. (c) Two triangles associated with a $\mathrm{CV}$ face. (d) A boundary CV. 
where $F_{k}$ and $A_{k}$ are the midpoint and the length of the $k$ th control volume face respectively. The term $(K \nabla \phi) \cdot \hat{\mathbf{n}}$ can be written as $\nabla \phi \cdot\left(K^{T} \hat{\mathbf{n}}\right)$ and hence, the above equation becomes

$$
\begin{gathered}
\rho C_{p} \frac{\delta V_{P}}{\delta t}\left(\phi_{P}^{(n+1)}-\phi_{P}^{(n)}\right)-\lambda \sum_{k=1}^{N p}\{(\nabla \phi) \cdot \mathbf{w}\}_{F_{k}}^{(n+1)} A_{k} \\
-(1-\lambda) \sum_{k=1}^{N p}\{(\nabla \phi) \cdot \mathbf{w}\}_{F_{k}}^{(n)} A_{k} \simeq 0
\end{gathered}
$$

where $\mathbf{w}=K^{T} \hat{\mathbf{n}}$. The parameter $\lambda=1$ gives a fully implicit scheme, $\lambda=0$ leads to a fully explicit scheme and $\lambda=\frac{1}{2}$ provides a second order scheme in time. The parameter $\lambda$ is set to 1 for this study.

\subsection{Flux decomposition using vectors (FDV)}

Figures 2(a) and 2(b) show the vectors associated with the flux decomposition at a control volume face. The unit vectors $\hat{\mathbf{n}}_{k}$ and $\hat{\mathbf{u}}_{k}$ are perpendicular to each other. The vector $\mathbf{v}_{k}$ that joins the points $P$ and $N_{k}$ is perpendicular to $\hat{\mathbf{t}}_{k}$.

The vector $\mathbf{w}_{k}=K^{T} \hat{\mathbf{n}}_{k}$ can be decomposed using the two vector equations

$$
\mathbf{v}=(\mathbf{v} \cdot \hat{\mathbf{u}}) \hat{\mathbf{u}}+(\mathbf{v} \cdot \hat{\mathbf{n}}) \hat{\mathbf{n}} \quad \text { and } \quad \mathbf{w}=(\mathbf{w} \cdot \hat{\mathbf{u}}) \hat{\mathbf{u}}+(\mathbf{w} \cdot \hat{\mathbf{n}}) \hat{\mathbf{n}}
$$

where subscript $k$ is suppressed for clarity. Thus,

$$
\mathbf{w}=(\mathbf{w} \cdot \hat{\mathbf{u}}) \hat{\mathbf{u}}+(\mathbf{w} \cdot \hat{\mathbf{n}}) \frac{\mathbf{v}-(\mathbf{v} \cdot \hat{\mathbf{u}}) \hat{\mathbf{u}}}{\mathbf{v} \cdot \hat{\mathbf{n}}}=\frac{\mathbf{w} \cdot \hat{\mathbf{n}}}{\mathbf{v} \cdot \hat{\mathbf{n}}} \mathbf{v}+\left\{\mathrm{w} \cdot \hat{\mathbf{u}}-\mathbf{w} \cdot \hat{\mathbf{n}} \frac{\mathbf{v} \cdot \hat{\mathbf{u}}}{\mathbf{v} \cdot \hat{\mathbf{n}}}\right\} \hat{\mathbf{u}} .
$$



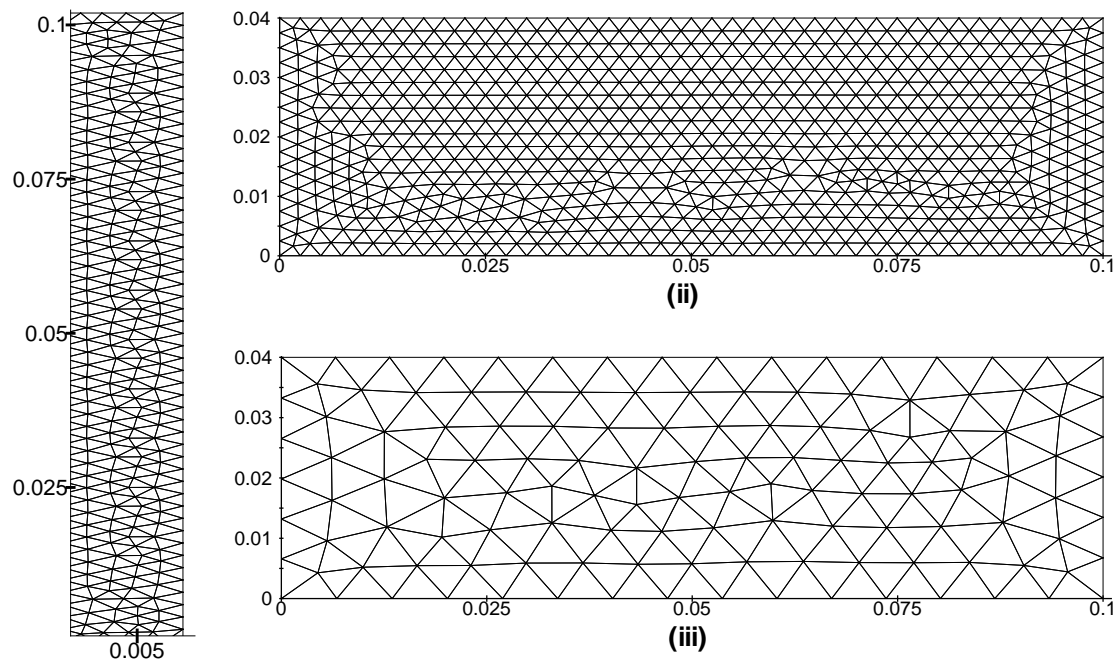

(ii)

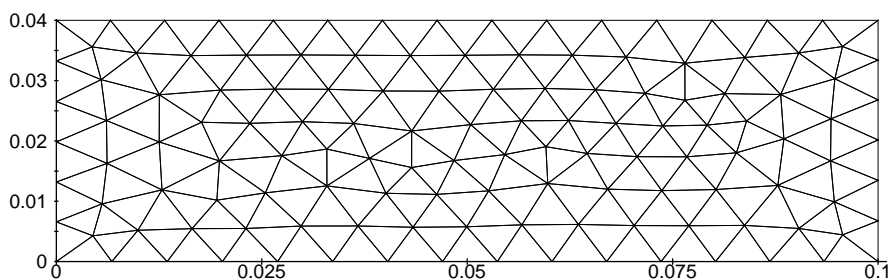

(iii)

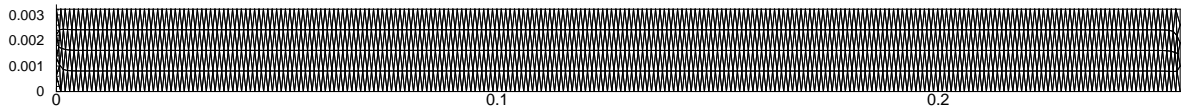

(iv)

Figure 3: Triangular meshes used for the simulations: (i) created on transformed domain, used for Case 2 - 328 nodes. (ii) created on real domain - 809 nodes. (iii) created on real domain - 131 nodes. (iv) created on transformed domain, used for Case 1 - 1284 nodes. 
Equation (6) can be used to decompose $\nabla \phi \cdot \mathbf{w}=\nabla \phi \cdot\left(K^{T} \hat{\mathbf{n}}\right)$ in terms of $\mathbf{v}$ and $\hat{\mathbf{u}}$ as follows:

$$
(\nabla \phi \cdot \mathbf{w})_{F_{k}}=\frac{\mathbf{w} \cdot \hat{\mathbf{n}}}{\mathbf{v} \cdot \hat{\mathbf{n}}}(\nabla \phi \cdot \mathbf{v})_{F_{k}}+\left\{\mathbf{w} \cdot \hat{\mathbf{u}}-\mathbf{w} \cdot \hat{\mathbf{n}} \frac{\mathbf{v} \cdot \hat{\mathbf{u}}}{\mathbf{v} \cdot \hat{\mathbf{n}}}\right\}(\nabla \phi \cdot \hat{\mathbf{u}})_{F_{k}} .
$$

Assuming the points $F_{k}$ and $R_{k}$ are sufficiently close, the following equations are obtained:

$$
(\nabla \phi \cdot \mathbf{v})_{F_{k}} \simeq \phi_{N_{k}}-\phi_{P}
$$

and

$$
(\nabla \phi \cdot \hat{\mathbf{u}})_{F_{k}} \simeq \alpha_{k}(\nabla \phi \cdot \hat{\mathbf{u}})_{P}+\left(1-\alpha_{k}\right)(\nabla \phi \cdot \hat{\mathbf{u}})_{N_{k}}
$$

where $\alpha_{k}=\frac{R N}{P N}$. Substitution of equations (8) and (9) into (7) gives the following expression for the flux at the control volume face:

$$
\begin{aligned}
& \{(K \nabla \phi) \cdot \hat{\mathbf{n}}\}_{F_{k}}^{(n+1)} \simeq \frac{\mathbf{w} \cdot \hat{\mathbf{n}}}{\mathbf{v} \cdot \hat{\mathbf{n}}}\left(\phi_{N_{k}}^{(n+1)}-\phi_{P}^{(n+1)}\right) \\
& +\left\{\mathbf{w} \cdot \hat{\mathbf{u}}-\mathbf{w} \cdot \hat{\mathbf{n}} \frac{\mathbf{v} \cdot \hat{\mathbf{u}}}{\mathbf{v} \cdot \hat{\mathbf{n}}}\right\}\left[\alpha_{k}(\nabla \phi \cdot \hat{\mathbf{u}})_{P}+\left(1-\alpha_{k}\right)(\nabla \phi \cdot \hat{\mathbf{u}})_{N_{k}}\right] .
\end{aligned}
$$

The gradients at the node points are calculated using the following approximation techniques discussed in $[1,2,6]$.

(i) GRF:

$$
(\nabla \phi)_{P} \simeq \frac{1}{\delta V_{P}} \sum_{k=1}^{p}\left\{\alpha_{k} \phi_{P}+\left(1-\alpha_{k}\right) \phi_{N_{k}}\right\} \hat{\mathbf{n}}_{k} A_{k} .
$$

(ii) GMF2:

$$
\begin{aligned}
& (\nabla \phi)_{P} \simeq \frac{\Lambda_{P_{b}}^{-1}}{\delta V_{P}} \sum_{k=1}^{p}\left\{\alpha_{k} \phi_{P}+\left(1-\alpha_{k}\right) \phi_{N_{k}}+\left(1-\alpha_{k}\right)(\nabla \phi)_{N_{k}} \cdot \delta \mathbf{x}_{k}\right\} \hat{\mathbf{n}}_{k} A_{k} \\
& \text { where } \Lambda_{P_{b}}=\left[I-\frac{1}{\delta V_{P}} \sum_{k=1}^{p} \alpha_{k} A_{k} \hat{\mathbf{n}}_{k}\left(\delta \mathbf{x}_{k}\right)^{T}\right] .
\end{aligned}
$$


(iii) Least squares gradient reconstruction:

$$
\begin{aligned}
& \left(\begin{array}{cc}
\sum_{k=1}^{p} w_{k} \Delta x_{k}^{2} & \sum_{k=1}^{p} w_{k} \Delta x_{k} \Delta y_{k} \\
\sum_{k=1}^{p} w_{k} \Delta x_{k} \Delta y_{k} & \sum_{k=1}^{p} w_{k} \Delta y_{k}^{2}
\end{array}\right)\left(\begin{array}{l}
\frac{\partial \phi}{\partial x} P \\
\frac{\partial \phi}{\partial y_{P}}
\end{array}\right) \\
& =\left(\begin{array}{c}
\sum_{k=1}^{p} w_{k} \Delta x_{k}\left(\phi_{N_{k}}-\phi_{P}\right) \\
\sum_{k=1}^{p} w_{k} \Delta y_{k}\left(\phi_{N_{k}}-\phi_{P}\right)
\end{array}\right)
\end{aligned}
$$

where $w_{k}=\left\|\mathbf{v}_{k}\right\|^{-c}$ and $\mathbf{v}_{k}=P \vec{N}_{k}=\left(\Delta x_{k}, \Delta y_{k}\right)$, with: $c=0$, LS; $c=1$, WLSID1; and $c=2$, WLSID2.

\subsubsection{Boundary conditions}

To obtain the cross diffusion terms for the boundary control volumes (see Figure 2(d)) the gradients at the boundary nodes need to be approximated. These gradients can be estimated using the function values at connected boundary nodes, together with boundary flux information. Suppose the gradient at node $P$ is to be evaluated and consider Figure 2(d), which shows a typical cluster of boundary control volumes at $x=0$. Using the boundary conditions it is possible to write, for the orthotropic case,

$$
\frac{\partial \phi}{\partial x}=\frac{h_{w}}{k_{x x}}\left(\phi_{P}-\phi_{w}\right) .
$$

The gradient component in the direction $y$ is written as

$$
\frac{\partial \phi}{\partial y} \simeq \frac{\left(\phi_{N}-\phi_{P}\right)}{\|\overrightarrow{P N}\|} \quad \text { or } \quad \frac{\partial \phi}{\partial y} \simeq \frac{\left(\phi_{N}-\phi_{O}\right)}{\|\overrightarrow{O N}\|}
$$


Therefore, the gradient at the point $P$ is approximated as, for example,

$$
(\nabla \phi)_{P} \simeq \frac{h_{w}}{k_{x x}}\left(\phi_{P}-\phi_{w}\right) \vec{i}+\frac{\left(\phi_{N}-\phi_{P}\right)}{\|\overrightarrow{P N}\|} \vec{j}
$$

Note that at the corner point $O$ the gradient is approximated as

$$
(\nabla \phi)_{O} \simeq \frac{h_{w}}{k_{x x}}\left(\phi_{O}-\phi_{w}\right) \vec{i}+\frac{h_{s}}{k_{y y}}\left(\phi_{O}-\phi_{s}\right) \vec{j}
$$

because there are two boundary conditions available.

To approximate the gradient on a face, say, on the face $F$ in Figure 2(d), the geometrical average can be used when the gradients for the points $P$ and $N$ are available. All of these calculations are carried out explicitly using the function values at time step $n$, as the function values are not available at time $(n+1) \delta t$.

\subsection{Hybrid CV-FE formulation}

The complete details of the hybrid control volume finite element method may be found in $[6,11]$. Here only the main results are summarised. After some manipulations the total flux $\nabla \phi \cdot \mathbf{w}_{k} A_{k}=$ $\nabla \phi \cdot\left(K^{T} \hat{\mathbf{n}}_{k}\right) A_{k}$, through the face $k$ is

$$
\nabla \phi \cdot\left(K^{T} \hat{\mathbf{n}}_{k}\right) A_{k} \simeq\left(K^{T} \hat{\mathbf{n}}_{k}^{(a)}\right)^{T} G_{k}^{(a)} \mathbf{d} \phi_{k}^{(a)} A_{k}^{(a)}+\left(K^{T} \hat{\mathbf{n}}_{k}^{(b)}\right)^{T} G_{k}^{(b)} \mathbf{d} \phi_{k}^{(b)} A_{k}^{(b)}
$$

where, for example,

$$
G_{k}^{(a)}=\left(\begin{array}{cc}
\frac{\partial}{\partial x} M_{N_{k}}^{(a)} & \frac{\partial}{\partial x} M_{N_{k a}}^{(a)} \\
\frac{\partial}{\partial y} M_{N_{k}}^{(a)} & \frac{\partial}{\partial y} M_{N_{k a}}^{(a)}
\end{array}\right) \quad \text { and } \quad \mathbf{d} \phi_{k}^{(a)}=\left(\begin{array}{c}
\phi_{N_{k}}-\phi_{P} \\
\phi_{N_{k a}}-\phi_{P}
\end{array}\right) .
$$


Thus, replacing the term $(\nabla \phi \cdot \mathbf{w})_{F_{k}} A_{k}$ in equation (5) (with $\lambda=1$ ) by equation (11) it follows that

$$
\begin{aligned}
& \rho C_{p} \frac{\delta V_{P}}{\delta t}\left(\phi_{P}^{(n+1)}-\phi_{P}^{(n)}\right)-\sum_{k=1}^{p}\left\{\left(K^{T} \hat{\mathbf{n}}_{k}^{(a)}\right)^{T} G_{k}^{(a)}\left(\mathbf{d} \phi_{k}^{(a)}\right)^{(n+1)} A_{k}^{(a)}\right. \\
&\left.+\left(K^{T} \hat{\mathbf{n}}_{k}^{(b)}\right)^{T} G_{k}^{(b)}\left(\mathbf{d} \phi_{k}^{(b)}\right)^{(n+1)} A_{k}^{(b)}\right\} \simeq 0
\end{aligned}
$$

where all off diagonal terms have been treated implicitly.

\subsubsection{Boundary Conditions}

At the boundary control volumes (see again Figure 2(d)), the value of the function at the boundary point $P$ is assumed to be the same as that of the boundary surfaces and all discrete quantities are calculated there. If a control volume face coincides with a boundary then the above equations are altered by setting the flux through that face equal to the boundary flux, evaluated at point $P$, multiplied by the length of the boundary control volume face. At a boundary control volume the discretised equations will take the following form:

$$
\begin{aligned}
& \rho C_{p} \frac{\delta V_{P}}{\delta t}\left(\phi_{P}^{(n+1)}-\phi_{P}^{(n)}\right)-\sum_{k=1}^{p} \zeta_{k}\left\{\left(K^{T} \hat{\mathbf{n}}_{k}^{(a)}\right)^{T} G_{k}^{(a)}\left(\mathbf{d} \phi_{k}^{(a)}\right)^{(n+1)} A_{k}^{(a)}\right\} \\
& -\sum_{k=1}^{p} \eta_{k}\left\{\left[\left(K^{T} \hat{\mathbf{n}}_{k}^{(b)}\right)^{T} G_{k}^{(b)}\left(\mathbf{d} \phi_{k}^{(b)}\right)^{(n+1)} A_{k}^{(b)}\right\}\right. \\
& -\sum_{b=1}^{N_{b}} h_{b}\left(\phi_{b}-\phi_{P}^{(n+1)}\right) A_{b} \simeq 0
\end{aligned}
$$


where $N_{b}$ is the number of boundary control volume faces and $\zeta_{k}$ (or $\eta_{k}$ ) is zero if triangle $A$ (or $B$ ) does not exist, or one if $A$ (or $B$ ) exists for a given CV face (see Figure 2(c)).

\section{Transformation into an Isotropic Model}

Using the scalings

$$
X=\frac{x}{\sqrt{k_{x x}}} \text { and } Y=\frac{y}{\sqrt{k_{y y}}},
$$

equation (1) is transformed into an isotropic model,

$$
\nabla^{\prime} \cdot\left(\nabla^{\prime} \phi\right)=\rho C_{p} \frac{\partial \phi}{\partial t}, \quad 0 \leq X \leq \frac{L}{\sqrt{k_{x x}}}, \quad 0 \leq Y \leq \frac{M}{\sqrt{k_{y y}}}, \quad t>0,
$$

where

$$
\nabla^{\prime}=\left(\frac{\partial}{\partial X}, \frac{\partial}{\partial Y}\right)
$$

The boundary conditions are changed to

$$
\left(K^{\prime} \nabla^{\prime} \phi\right) \cdot \hat{\mathbf{n}}_{b}=h_{b}\left(\phi_{s}-\phi\right), \quad \text { at boundary } S_{b}, \quad t>0,
$$

where $K^{\prime}=\operatorname{diag}\left(\sqrt{k_{x x}}, \sqrt{k_{y y}}\right)$. Equation (16) can be solved using the finite volume techniques discussed in [6] with the control volume meshes created on the transformed domain. Clearly, very strong anisotropy ratios (i.e. $k_{x x}: k_{y y}=p: q$ ) will make the transformed domain thinner in the $y$ direction to the extent of being impractical, or difficult, when the ratio $p: q$ is very large or very small, see Figures 3(i) and 3(iv). This issue needs to be considered when using this method to resolve the problem numerically. 
TABlE 1: Comparison of errors using the formula: RMSE $=$ $\sqrt{\frac{1}{N} \sum_{i=1}^{N}\left(\phi_{i}^{n}-\phi_{i}^{e}\right)^{2}}$ where $\phi^{e}$ is the exact solution, $\phi^{n}$ is the numerical result and $N$ is the number of node points. For descriptions of meshes see Figure 3.

\begin{tabular}{lllllrl}
\hline & \multicolumn{3}{c}{ Case 1: $k_{x x}: k_{y y}=1: 1000$} & \multicolumn{2}{c}{ Case $2: k_{x x}: k_{y y}=1000: 1$} \\
RMSE & Mesh (ii) & Mesh (iii) & Mesh (iv) & Mesh (ii) & Mesh (iii) & Mesh (i) \\
& $N=809$ & $N=131$ & $N=1284$ & $N=809$ & $N=131$ & $N=328$ \\
\hline GRF & 2.812477 & 5.882836 & 0.015703 & 0.803156 & 1.223945 & 0.026352 \\
GMF2 & 2.683718 & 5.680837 & 0.015708 & 0.804600 & 1.223406 & 0.026644 \\
LS & 2.680167 & 5.728777 & 0.015706 & 0.828324 & 1.225261 & 0.026618 \\
WLSID1 & 2.679774 & 5.658741 & 0.015708 & 0.819475 & 1.216931 & 0.026616 \\
WLSID2 & 2.679926 & 5.584183 & 0.015714 & 0.812039 & 1.222791 & 0.026618 \\
Hybrid & 1.446686 & 2.359577 & 0.012931 & 0.753764 & 5.328590 & 0.012708 \\
\hline
\end{tabular}

\section{$5 \quad$ Numerical Results}

To test the techniques discussed throughout Sections 3 and 4, a wood like material is used. In this case all faces are subjected to the mixed boundary conditions described in Section 1. Throughout the tests a material with the physical properties: $L=0.1 \mathrm{~m}$, $M=0.04 \mathrm{~m}, h_{b}=10 \mathrm{~W} / \mathrm{m}^{2} / \mathrm{K}, \rho=600 \mathrm{~kg} / \mathrm{m}^{3}$ and $C_{p}=1.6886 \times$ $10^{3} \mathrm{~J} / \mathrm{kg} / \mathrm{K}$ is used with $\phi_{0}=30^{\circ} \mathrm{C}$ and $\phi_{s}=140^{\circ} \mathrm{C}$. The values $k_{x x}=0.154 \mathrm{~W} / \mathrm{m} / \mathrm{K}, k_{y y}=154 \mathrm{~W} / \mathrm{m} / \mathrm{K}$ for Case 1 (or $k_{x x}=$ $154 \mathrm{~W} / \mathrm{m} / \mathrm{K}, k_{y y}=0.154 \mathrm{~W} / \mathrm{m} / \mathrm{K}$ for Case 2 ) are used to obtain the results presented here. The time step, $\delta t$, used for the simulations presented here is one second and the results shown are obtained after 1000 seconds. The meshes used throughout the analysis are shown in Figure 3. These meshes were created using the mesh generator, EasyMesh, developed by Niceno [9] and the centroids of the triangles are used as element centres for the construction of the control volumes.

Table 1 summarises the important findings of this work and the 

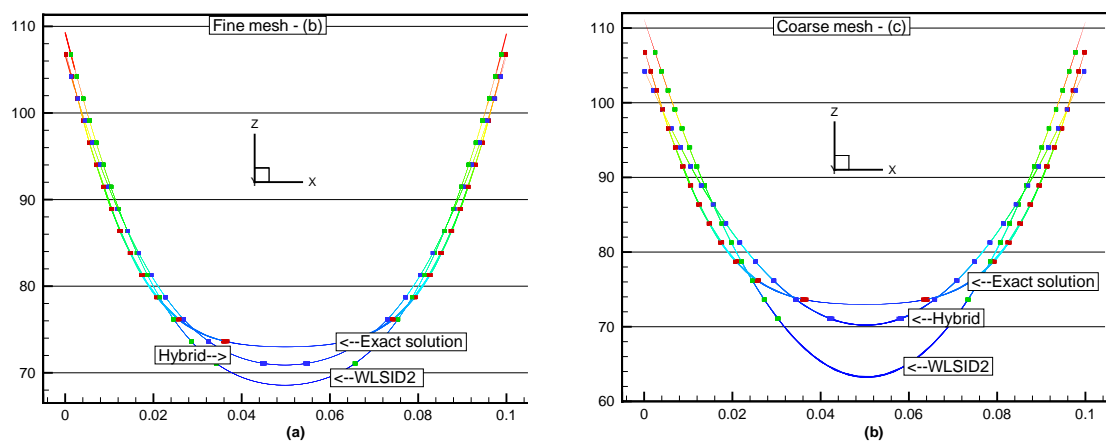

FiguRE 4: Results of the untransformed problem: (a) on mesh (ii) for the ratio $k_{x x}: k_{y y}=1: 1000$ (Case 1) and (b) on mesh (iii) for the ratio $k_{x x}: k_{y y}=1: 1000$ (Case 1$)$.
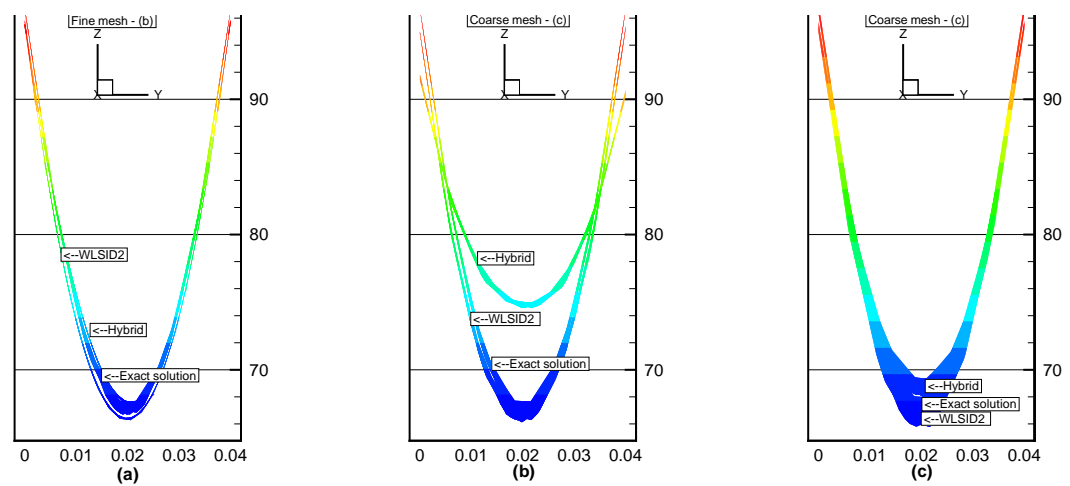

FiguRE 5: Results of the untransformed problem: (a) on mesh (ii) for the ratio $k_{x x}: k_{y y}=1000: 1$ (Case 2), (b) on mesh (iii) for the ratio $k_{x x}: k_{y y}=1000: 1$ (Case 2) and (c) on mesh (iii) for the ratio $k_{x x}: k_{y y}=100: 1$. 


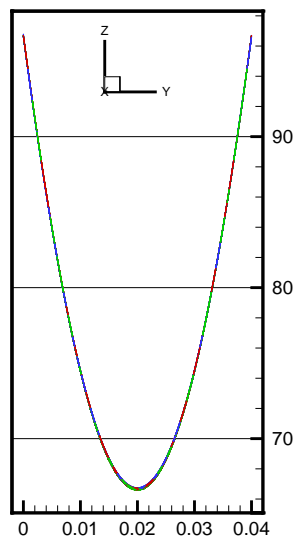

(a)

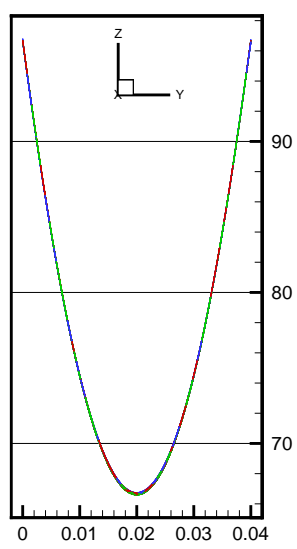

(b)

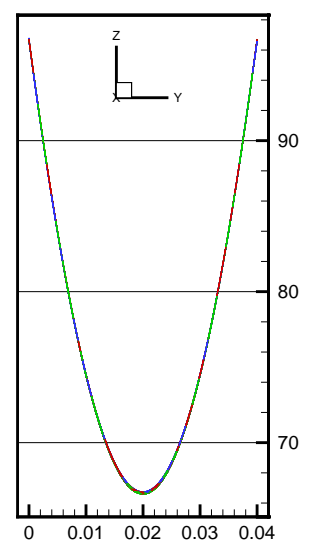

(c)

FiguRE 6: Results obtained using the method of transformation on mesh (i): (a) Exact, (b) WILSD2 and (c) Hybrid for the ratio $k_{x x}: k_{y y}=1000: 1$ (Case 2). 
results for some selected simulations have been exhibited in Figures 4 and 5 .

The main observation to be found from Table 1 and the supporting graphs is that the use of shape functions (hybrid method) provides more accurate results than the other methods for both Case 1 and Case 2, except for Case 2 on the coarse mesh (Figure 3(iii)). It is worthwhile to observe that the hybrid method produces misleading and inaccurate results for Case 2 on the coarse mesh, while it has very good agreement with the exact solution on the fine mesh (Figure 3(ii)).

Results for the cases where $k_{x x}: k_{y y}=1: 10,1: 100,10: 1$, $100: 1$ were obtained for the untransformed model and found to have good agreement with the exact solutions on both the fine and coarse meshes, and therefore only a selected case, $k_{x x}: k_{y y}=100: 1$, is depicted here in Figure 5(c).

On the other hand, the use of the least squares method provides good results for Case 2 on both the fine and coarse meshes and that method fails to give good results for Case 1, especially on the coarse mesh for the original problem. However, for Case 1, the hybrid method provides reasonable results on both meshes.

Table 1 also shows that the four gradient approximation techniques, namely GRF, GMF2, LS, WLSID1 and WLSID2, produce approximately the same results on each mesh for all cases and therefore only the results produced by the least squares method are exhibited in Figures 4 and 5 to compare with the exact solution and the results from the hybrid method. Observe in Figure 4 that the numerical techniques discussed here for the untransformed problem are unable to produce good results for Case 1 on both meshes. The inaccuracy of the hybrid technique on a coarse mesh for Case 2 is clearly shown in Figure 5(b). Note, however, that all of the methods provide very 
good results for both cases after transformation into an isotropic model (see Table 1 and Figure 6).

\section{Conclusions}

The work revealed that the use of existing gradient approximation techniques for simulating orthotropic transport problems produces reasonable results on uniform, fine meshes. However in general, the use of fine meshes cannot be recommended because the computational cost will be high, especially in three-dimensional cases. To obtain accurate results on coarse meshes, it is necessary to use a higher order gradient approximation technique to calculate the flux at the control volume faces. The decomposition technique used for the flux also plays a major role in the finite volume scheme. Currently, the authors are developing higher order fully implicit finite volume methods to rectify this problem. The transformation of the orthotropic model into an isotropic model produces very good results using the available gradient approximation techniques. However, such transformations may not be possible to use for coupled equations or problems where the material properties are continually changing over time. In this case it would be computationally inefficient to continually transform and re-mesh the domain throughout the processing in time.

Acknowledgments: The first author acknowledges the financial support provided by the Queensland University of Technology under the IPRS scholarship program and the leave granted for the studies from the University of Ruhuna, Sri Lanka. 


\section{References}

[1] T. J. Barth, Aspects of Unstructured Grids and Finite-Volume Solvers for the Euler and Navier-Stokes Equations, in: Lecture Notes Presented at the VkI Lecture Series 1994-05, Feb. 1994. C452

[2] N. Croft, Unstructured Mesh - Finite Volume Algorithms for Swirling, Turbulent, Reacting Flows, PhD. Thesis, University of Greenwich, London, UK, 1998. C445, C452

[3] I. Demirdžić and S. Muzaferija, Numerical method for coupled fluid flow, heat transfer and stress analysis using unstructured moving meshes with cells of arbitrary topology, Compt. Meth. Appl. Mech. Eng., Vol. 125, pp. 235-255, 1995. C445

[4] I. Faille, A control volume method to solve an elliptic equation on a two-dimensional irregular meshing, Compt. Meth. Appl. Mech. Eng., Vol. 100, 1992. C445

[5] F. Hermeline, A Finite Volume Method for the Approximation of Diffusion Operators on Distorted Meshes, Journal of Computational Physics, Vol. 160, pp. 481-499, 2000. C445

[6] P. A. Jayantha and I. W. Turner, A Comparison of Gradient Approximations for use in Finite Volume Computational Models for Two-Dimensional Diffusion Equations, Numerical Heat Transfer, Part B: Fundamentals, Vol. 40(5), pp. 367-390, 2001. C447, C452, C454, C456

[7] A. Jennings and J. J. McKeown, Matrix Computation, 2nd ed., John Wiley \& Sons Ltd, New York, 1992. C448 
[8] J. Y. Murthy and S. R. Mathur, Computation of Anisotropic Conduction using Unstructured Meshes, Journal of heat Transfer, Vol. 120, pp. 583-591, 1998. C445

[9] B. Niceno, "EasyMesh" (Version 1.4), http://www-dinma. univ.trieste.it/ nirftc/research/easymesh/. C457

[10] M. N. Özişik, Heat Conduction, 1st ed., Wiley, New York, 1980. C447

[11] P. Perré and I. Turner, TransPore: A Generic Heat and Mass Transfer Computational Model for Understanding and Visualising The Drying Of Porous Media, Invited paper, Drying Technology Journal, Vol. 17(7), 1273-1289, 1999. C454 